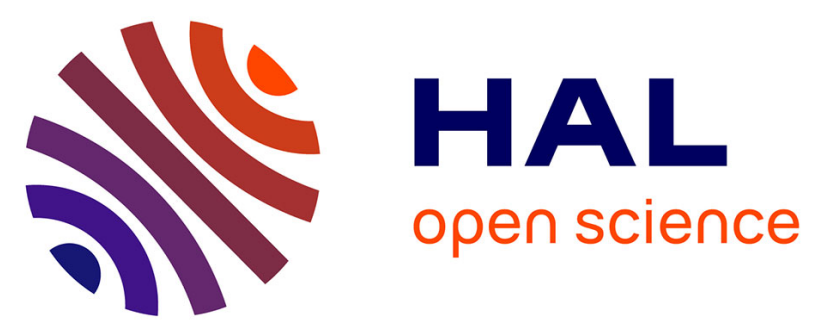

\title{
An Innovative Nonlinear Filter for Radar Kinematic Estimation of Maneuvering Targets in 2D
}

Marion Pilté, Silvère Bonnabel, Frédéric Barbaresco

\section{To cite this version:}

Marion Pilté, Silvère Bonnabel, Frédéric Barbaresco. An Innovative Nonlinear Filter for Radar Kinematic Estimation of Maneuvering Targets in 2D. 2017 18th International Radar Symposium (IRS), Jun 2017, Prague, Czech Republic. 10.23919/IRS.2017.8008156 . hal-01692475

HAL Id: hal-01692475

https://hal-mines-paristech.archives-ouvertes.fr/hal-01692475

Submitted on 25 Jan 2018

HAL is a multi-disciplinary open access archive for the deposit and dissemination of scientific research documents, whether they are published or not. The documents may come from teaching and research institutions in France or abroad, or from public or private research centers.
L'archive ouverte pluridisciplinaire HAL, est destinée au dépôt et à la diffusion de documents scientifiques de niveau recherche, publiés ou non, émanant des établissements d'enseignement et de recherche français ou étrangers, des laboratoires publics ou privés. 


\title{
An Innovative Nonlinear Filter for Radar Kinematic Estimation of Maneuvering Targets in 2D
}

\author{
Marion Pilté $^{*, * *}$, Silvère Bonnabel ${ }^{*}$, Frédéric Barbaresco** \\ *MINES ParisTech, PSL Research University, Centre for Robotics \\ 60 Bd St Michel, 75006 Paris, FRANCE \\ email: marion.pilte@mines-paristech.fr, silvere.bonnabel@mines-paristech.fr \\ ** Thales Air Systems \\ Voie Pierre Gilles de Gennes, hameau de Roussigny, 91120 Limours, FRANCE \\ email: frederic.barbaresco@ thalesgroup.com

\begin{abstract}
In this paper we consider the problem of tracking a target in a $2 D$ space whose model is based on a constant velocity assumption (up to a process noise) in the FrenetSerret frame (or intrinsic coordinates). This model is particularly suited to straight lines and coordinated turn motions. Then, we adapt the Invariant Extended Kalman Filter (I$E K F)$, as in [1], a novel variant of the EKF on Lie groups, to suit this dynamical model. Numerical experiments inspired by real fighter trajectories confirm the validity of our approach.
\end{abstract}

\section{Introduction}

In radar tracking, assuming we have a proper plot-track association, the aim of filtering is to recover the velocity of the target, essentially to guide the beam of the radar at the next step, see for example [2]. At the same time, the position must be estimated as well (i.e., filtering the noisy measurements). The challenge of filter design is two fold: first a probabilistic model of the target evolution must be chosen with the process noise assessed, and then a non-linear estimator must be designed. In the present paper, we address both challenges.

Regarding the choice of a model, recent work [3] has advocated the case of intrinsic coordinates based models. Indeed, it looks intuitive that the commands of the plane be piecewise constant in a frame attached to the target (the Frenet-Serret frame) although it is not a widespread approach in the literature. For instance, the coordinated turn model can be easily expressed with intrinsic coordinates. In this paper, we opt for such a model.

Then, there are various filters to perform nonlinear estimation (Extended Kalman Filter (EKF) [4], Unscented Kalman Filter (UKF) [5], particle filters [6], Interacting Multiple Model (IMM) filters [7] which is the most widespread, but which relies on banks of EKFs), see [8] for a general view on nonlinear filtering. The tracking of maneuvering targets is a well-known problem and extensions of the Kalman filter, or Kalman-Bucy filter to nonlinear target evolution equations have been widely studied, see for example [?] or [9]. However convergence properties are rarely shown, expecially with the most widely used extension of the Kalman filter, the 
Extended Kalman Filter (EKF). This filter is not stable when the system is highly nonlinear, see [10]. Convergence properties are derived assuming the error is sufficiently small, for example [11] or [12], but this assumption is not valid for highly maneuvering targets. Recently, a methodology for extended kalman filtering emerged, namely the Invariant Extended Kalman Filter (IEKF), see e.g. [13]. This method guarantees convergence for some theoretically pure cases, when the model perfectly fits the theory. Such convergence results do not exist for the EKF. Moreover, the IEKF is suited to coordinates related to the Frenet frame. We extend the method to a case that is not encompassed in the framework of [13].

This paper is organized as follows: Section 2 briefly recalls the equations of the EKF, Section 3 explains the principle of the IEKF, based on the description in [14], for some known parameters and cartesian measurements, Section 4 extends the use of this filter to the case where the angular velocity and the norm of the velocity are unknown and to polar measurements. Section 5 provides a radar application example inspired by a real fighter trajectory, and a comparison between the EKF and the IEKF for a given trajectory.

\section{The Extended Kalman Filter}

In this section, we introduce the EKF equations in a general case, to point out why this filter is not stable. More detailed equations can be found in [4]. The evolution model is supposed to be nonlinear. $X_{n}$ is the state of the system at time instant $n, f$ and $h$ are nonlinear functions, $w_{n}$ and $v_{n}$ are gaussian white noises. In the general case, the evolution is described by (1).

$$
\left\{\begin{aligned}
X_{n} & =f\left(X_{n-1}, w_{n}\right) \\
Y_{n} & =h\left(X_{n}\right)+v_{n}
\end{aligned}\right.
$$

The propagation phase of the algorithm is $\hat{X}_{n \mid n-1}=f\left(\hat{X}_{n-1 \mid n-1}, 0\right)$, and the error is defined by $e_{n \mid n-1}=X_{n}-\hat{X}_{n \mid n-1}, \quad e_{n-1 \mid n-1}=X_{n-1}-\hat{X}_{n-1 \mid n-1}$. The linearization of this error takes the following form:

$$
e_{n \mid n-1}=\frac{\partial}{\partial X} f\left(\hat{X}_{n-1 \mid n-1}, 0\right) e_{n-1 \mid n-1}+\frac{\partial}{\partial w} f\left(\hat{X}_{n-1 \mid n-1}, 0\right) w_{n}
$$

We define $F_{n}$ and $G_{n}$, the matrices used for the Riccati equation, so that

$$
F_{n} e_{n-1 \mid n-1}+G_{n} w_{n}=e_{n \mid n-1}
$$

The problem is that these matrices depend on the predicted state $\hat{X}_{n \mid n-1}$.

For the update phase of the algorithm, we use the linearization of the measurement function $H_{n}=\frac{\partial}{\partial X} h\left(\hat{X}_{n \mid n-1}\right)$. We then use a standard Kalman filter to compute the error estimate: the Kalman gain $K_{n}$ can be derived thanks to the Riccati equation. For the derivation of the Kalman gain, see for instance [15]. As $K_{n} z_{n}$ (with $z_{n}=Y_{n}-h\left(\hat{X}_{n \mid n-1}\right)$ ) is an estimate of $e_{n \mid n}$, we have $\hat{X}_{n \mid n}=\hat{X}_{n \mid n-1}+K_{n} z_{n}$. 


\section{The Invariant Extended Kalman Filter}

In this section, we recall the equations of the Invariant Extended Kalman Filter (IEKF) as presented in [13] on a specific example, that we will broaden in the next section. We do not give any proof of convergence, they can be found in [13]. We assume here the evolution model of the system is described with equations (4). It is the equations of the movement of a non-holonomous car, which means the car cannot slide. The evolution is here in continuous time. However, the measures are in discrete time (5).

$$
\begin{gathered}
\frac{d}{d t} \theta_{t}=\omega_{t}+w_{t}^{\theta}, \frac{d}{d t} x_{t}^{(1)}=\left(u_{t}+w_{t}^{x}\right) \cos \left(\theta_{t}\right), \frac{d}{d t} x_{t}^{(2)}=\left(u_{t}+w_{t}^{x}\right) \sin \left(\theta_{t}\right) \\
Y_{n}=\left(x_{t_{n}}^{(1)}, x_{t_{n}}^{(2)}\right)^{T}+v_{n}
\end{gathered}
$$

$\theta_{t}$ is the direction of the object, $\left(x_{t}^{(1)}, x_{t}^{(2)}\right)$ its cartesian position. In this section, the angular velocity, $\omega_{t}$, and the norm of the velocity of the target, $u_{t}$ are supposed to be known. We shall see later how we can estimate these parameters. The measurement noise $v_{n}$ and the process noises $w_{t}^{\theta}$ and $w_{t}^{x}$ are white gaussian noises. In this paper, we will use this model to describe the movement of a maneuvering target in two dimensions.

The main idea of this method is to express the equations in matrix form, as in (7), to use the (left) invariance of the system. To do so, we introduce the state matrix:

$$
\begin{gathered}
\chi_{t}=\left(\begin{array}{ccc}
\cos \theta_{t} & -\sin \theta_{t} & x_{t}^{(1)} \\
\sin \theta_{t} & \cos \theta_{t} & x_{t}^{(2)} \\
0 & 0 & 1
\end{array}\right) \\
\left\{\begin{array}{c}
\frac{d \chi_{t}}{d t}=\chi_{t}\left(\nu_{t}+w_{t}\right) \\
Y_{n}=\left(\begin{array}{c}
x_{t_{n}}+v_{n} \\
1
\end{array}\right)=\chi_{t_{n}}\left(\begin{array}{c}
0_{2 \times 1} \\
1
\end{array}\right)+\left(\begin{array}{c}
v_{n} \\
0
\end{array}\right)
\end{array}\right.
\end{gathered}
$$

with $\nu_{t}=\left(\begin{array}{c}\omega_{t} \\ u_{t} \\ 0\end{array}\right)^{\wedge}=\left(\begin{array}{ccc}0 & -\omega_{t} & u_{t} \\ \omega_{t} & 0 & 0 \\ 0 & 0 & 0\end{array}\right)$ and $w_{t}=\left(\begin{array}{ccc}0 & -w_{t}^{\theta} & w_{t}^{x} \\ w_{t}^{\theta} & 0 & 0 \\ 0 & 0 & 0\end{array}\right)$. The notation $\left(\begin{array}{l}\omega \\ u_{1} \\ u_{2}\end{array}\right)^{\wedge}=$ $\left(\begin{array}{ccc}0 & -\omega & u_{1} \\ \omega & 0 & u_{2} \\ 0 & 0 & 0\end{array}\right)$, can be found in [16].

The space the state matrix lives in is called a matrix Lie group, namely $S E(2)$, the group of planar isometries. Elements of this group are written $G \in S E(2) \Longrightarrow G=\left(\begin{array}{cc}R(\theta) & x \\ 0_{1,2} & 1\end{array}\right)$ with $R(\theta)$ a rotation matrix in dimension 2. A Lie algebra is associated to each Lie group, it corresponds to the tangent space at the neutral element of the group. Here, the Lie algebra is $\mathfrak{s e}(2)$, and its elements are of the form $g \in \mathfrak{s e}(2) \Longrightarrow g=\left(\begin{array}{ccc}0 & -\theta & u_{1} \\ \theta & 0 & u_{2} \\ 0 & 0 & 0\end{array}\right),\left(\begin{array}{c}\theta \\ u_{1} \\ u_{2}\end{array}\right) \in \mathbb{R}^{3}$. 
The difference with the EKF is the definition of the error: it is called $\eta$, and it is a multiplication with a matricial inversion $\eta_{t}=\chi_{t}^{-1} \hat{\chi}_{t}$ and no longer a difference (which would be $\hat{\chi}_{t}-\chi_{t}$ ). The computations in [13] show that the linearized error $\left(\xi_{t} \in \mathbb{R}^{3}\right.$, such that $\left.\eta_{t} \approx I_{3}+\xi_{t}^{\wedge}\right)$ evolution is:

$$
\left\{\begin{aligned}
\frac{d}{d t} \xi_{t} & =-A_{t} \xi_{t}-\left(\begin{array}{c}
w_{t}^{\theta} \\
w_{t}^{x} \\
0
\end{array}\right) \\
\xi_{t_{n}}^{+} & =\xi_{t_{n}}-\tilde{K}_{n}\left[\left(0_{2,1}, I_{2}\right) \xi_{t}-R\left(\hat{\theta}_{t_{n}}\right)^{T} v_{n}\right]
\end{aligned}\right.
$$

if we let $A_{t}=\left(\begin{array}{ccc}0 & 0 & 0 \\ 0 & 0 & \omega_{t} \\ u_{t} & -\omega_{t} & 0\end{array}\right)$, and the gain $K_{n}=\tilde{K}_{n} \tilde{p}$ with $\tilde{p}=\left(I_{2}, 0_{2,1}\right) . K_{n}$ can be computed with the Riccati equation $\frac{d P_{t}}{d t}=A_{t} P_{t}+P_{t} A_{t}+\hat{Q}_{t}$, and $S_{n}=H P_{t_{n}} H^{T}+\hat{N}_{n}$, $K_{n}=P_{t_{n}} H^{T} S^{-1}, P_{t_{n}}^{+}=\left(I-L_{n} H\right) P_{t_{n}}$, with $H=\left(\begin{array}{ccc}0 & 1 & 0 \\ 0 & 0 & 1\end{array}\right), \hat{N}=R\left(\hat{\theta}_{t_{n}}\right) \operatorname{Cov}\left(v_{n}\right) R\left(\hat{\theta}_{t_{n}}\right)^{T}$ and $\hat{Q}_{t}=\operatorname{Cov}\left[\left(w_{t}^{\theta}, w_{t}^{x}, 0\right)^{T}\right]$.

Note that with isotropic measurement noise, i.e. $\operatorname{Cov}\left(v_{n}\right)=\alpha I_{2}$, the gain does not depend on the estimated state, and thus the filter presents some quite strong convergence properties. This is due to the fact that the evolution of the linearized error does not depend on the estimated state. For more information, see [13].

\section{Angular velocity and norm of the velocity estimation}

In the previous section, $\omega_{t}$ and $u_{t}$ were supposed to be known. For radar application this is obviously not the case. So we have to estimate these parameters. We include them in the state vector. Here we will not have the same guarantees of convergence as in the previous section, but the filter should still be quite stable since the dependance of the error on the predicted state is reduced to parameters derived from the position (it is only dependent on angular velocity and on the norm of the velocity, but not on the position itself). We keep the same notations as in Section 3. We deal with both linear and nonlinear measurements.

\subsection{Evolution equations and linearization}

We want to estimate $\omega$ and $u$ along with the position and the angle. The state vector is now $X_{t}=\left(\theta_{t}, x_{t}^{(1)}, x_{t}^{(2)}, \omega_{t}, u_{t}\right)^{T}$. The dynamics are as follows:

$$
\frac{d}{d t} \chi_{t}=\chi_{t}\left(\nu_{t}+w_{t}\right), \quad \frac{d}{d t} \kappa_{t}=\left(\begin{array}{c}
w_{t}^{\omega} \\
w_{t}^{u}
\end{array}\right)
$$

if we keep $\chi_{t}=\left(\begin{array}{ccc}\cos \theta_{t} & -\sin \theta_{t} & x_{t}^{(1)} \\ \sin \theta_{t} & \cos \theta_{t} & x_{t}^{(2)} \\ 0 & 0 & 1\end{array}\right)$ and if we let $\kappa_{t}=\left(\begin{array}{l}\omega_{t} \\ u_{t}\end{array}\right) . w_{t}^{\omega}$ and $w_{t}^{u}$ are white gaussian noises. 
The matricial form of the state is now

$$
\tilde{\chi}_{t}=\left(\begin{array}{ccccc}
\cos \theta_{t} & -\sin \theta_{t} & x_{t}^{(1)} & 0 & u_{t} \\
\sin \theta_{t} & \cos \theta_{t} & x_{t}^{(2)} & \omega_{t} & 0 \\
0 & 0 & 1 & 0 & 0 \\
0 & 0 & 0 & 1 & 0 \\
0 & 0 & 0 & 0 & 1
\end{array}\right):=\left(\begin{array}{c|cc}
\chi_{t} & 0 & u_{t} \\
\omega_{t} & 0 \\
\hline 0_{2 \times 3} & \multicolumn{2}{|c}{I_{2}}
\end{array}\right)
$$

Define the state error as $\tilde{\eta}_{t}=\tilde{\chi}_{t}^{-1} \hat{\tilde{\chi}}_{t}$. To linearize it, we have decomposed the state into two parts (as in (9)): $\chi_{t}$ and $\kappa_{t}$. The error is also decomposed into two parts: $\eta_{t}=\chi_{t}^{-1} \hat{\chi}_{t}$ and $\gamma_{t}=\hat{\kappa}_{t}-\kappa_{t}$.

We have the following equation for the evolution of the error $\eta_{t}$ :

$$
\frac{d}{d t} \eta_{t}=\eta_{t} \hat{\nu}_{t}-\nu_{t} \eta_{t}-w_{t} \eta_{t}
$$

To perform the linearisation, let $\mathbb{R}^{2} \ni \zeta_{t}=\left(\begin{array}{c}\hat{\omega}_{t} \\ \hat{u}_{t}\end{array}\right)-\left(\begin{array}{c}\omega_{t} \\ u_{t}\end{array}\right)$ the linearization of $\gamma_{t}$. Then $\nu_{t}=$ $\hat{\nu}_{t}-\left(\begin{array}{c}\zeta_{t} \\ 0\end{array}\right) \wedge$. The global linearized error is defined as $\tilde{\xi}_{t}=\left(\begin{array}{c}\xi_{t} \\ \zeta_{t}\end{array}\right)$ with $\xi_{t}$ the linearization of $\eta_{t}$.

Equation (11) becomes $\frac{d}{d t} \eta_{t}=\eta_{t} \hat{\nu}_{t}-\hat{\nu}_{t} \eta_{t}+\left[\left(\begin{array}{c}\zeta_{t} \\ 0\end{array}\right)^{\wedge}\right] \eta_{t}-w_{t} \eta_{t}$, and $\frac{d}{d t} \zeta_{t}=\left[\left(\begin{array}{c}w_{t}^{\omega} \\ w_{t}^{u} \\ 0\end{array}\right)^{\wedge}\right] \zeta_{t}$.

A mere analogy with the results of section II shows that the linearized equation writes:

$$
\frac{d}{d t} \xi_{t}=-A_{t} \xi_{t}+\left(\begin{array}{c}
\zeta_{t} \\
0
\end{array}\right)-\left(\begin{array}{c}
w_{t}^{\theta} \\
w_{t}^{x} \\
0
\end{array}\right)
$$

with $A_{t}$ defined as in (8). This gives:

$$
\frac{d}{d t} \tilde{\xi}_{t}=-\tilde{A}_{t} \tilde{\xi}_{t}-\left(\begin{array}{c}
w_{t}^{\theta} \\
w_{t}^{x} \\
0 \\
w_{t}^{\omega} \\
w_{t}^{v}
\end{array}\right)
$$

where $\tilde{A}_{t}=\left(\begin{array}{ccccc}0 & 0 & 0 & -1 & 0 \\ 0 & 0 & \hat{\omega}_{t} & 0 & -1 \\ \hat{u}_{t} & -\hat{\omega}_{t} & 0 & 0 & 0 \\ 0 & 0 & 0 & 0 & 0 \\ 0 & 0 & 0 & 0 & 0\end{array}\right)$ 


\subsection{Position measurements in cartesian coordinates}

In this paragraph, the measures are cartesian: $Y_{n}=\left(x_{t_{n}}^{(1)}, x_{t_{n}}^{(2)}\right)^{T}+v_{n}$. This gives the measurement matrix $\tilde{H}_{n}^{\text {cart }}=\left(\begin{array}{ccccc}0 & 1 & 0 & 0 & 0 \\ 0 & 0 & 1 & 0 & 0\end{array}\right)$. Applying standard Kalman equations to the obtained linearized model yields the following algorithm:

1. Propagation:

$$
\begin{gathered}
\frac{d}{d t} \hat{\theta}_{t}=\omega_{t}, \frac{d}{d t} \hat{x}_{t}=\left(\begin{array}{c}
\cos \hat{\theta}_{t} \\
\sin \hat{\theta}_{t}
\end{array}\right) u_{t}, \frac{d}{d t} \hat{\omega}_{t}=0, \frac{d}{d t} \hat{u}_{t}=0 \\
\frac{d}{d t} P_{t}=\tilde{A}_{t} P_{t}+P_{t} \tilde{A}_{t}+\hat{Q}_{t}
\end{gathered}
$$

2. Update:

$$
\begin{gathered}
K_{n}=P_{t_{n}} \tilde{H}_{n}^{\text {cart }^{T}}\left(\tilde{H}_{n}^{\text {cart }} P_{t_{n}} \tilde{H}_{n}^{\text {cart }}+\hat{N}\right)^{-1} \\
z_{n}=R\left(\hat{\theta}_{t_{n}}\right)^{T}\left(Y_{n}-\hat{x}_{t_{n}}\right) \\
e=K_{n} z_{n} \\
\left(\begin{array}{cc}
R\left(\hat{\theta}_{t_{n}}^{+}\right) & \hat{x}_{t_{n}}^{+} \\
0_{1,2} & 1
\end{array}\right)=\left(\begin{array}{cc}
R\left(\hat{\theta}_{t_{n}}\right) & \hat{x}_{t_{n}} \\
0_{1,2} & 1
\end{array}\right) \exp _{m}\left(e(1: 3)^{\wedge}\right), \hat{\omega}_{t_{n}}^{+}=\hat{\omega}_{t_{n}}+e(4), \hat{u}_{t_{n}}^{+}=\hat{u}_{t_{n}}+e(5)
\end{gathered}
$$

with $\exp _{m}$ the matrix exponential.

\subsection{Range and bearing measurements}

If the measures are expressed in range and bearing, called $r$ and $\alpha$ respectively, the measurement function then becomes nonlinear. Its expression is $Y_{n}=\left(r_{n}, \alpha_{n}\right)=h\left(x_{t_{n}}^{(1)}, x_{t_{n}}^{(2)}\right)=$ $\left(\sqrt{\left(x_{t_{n}}^{(1)}\right)^{2}+\left(x_{t_{n}}^{(2)}\right)^{2}}, \arctan \left(\frac{x_{t_{n}}^{(2)}}{x_{t_{n}}^{(1)}}\right)\right)$. In order to compute the Kalman gain, $\tilde{H}_{n}^{\text {pol }}$, the linearization of the function $h$ must be computed. $\tilde{H}_{n}^{\text {pol }}$ should only depend on $\hat{X}=\left(\begin{array}{c}\hat{\theta} \\ \hat{x}^{(1)} \\ \hat{x}^{(2)}\end{array}\right)$.

Simple computations show that

$$
Y_{n}-\hat{Y}_{n}=Y_{n}-h\left(x_{t_{n}}^{(1)}, x_{t_{n}}^{(2)}\right)=\tilde{H}_{n}^{\mathrm{pol}} \tilde{\xi}_{t_{n}}+O\left(\|\tilde{\xi}\|^{2}\right)
$$

and

$$
\tilde{H}_{n}^{\mathrm{pol}}=\nabla h_{\hat{x}} R(\hat{\theta})\left(\begin{array}{lllll}
0 & 1 & 0 & 0 & 0 \\
0 & 0 & 1 & 0 & 0
\end{array}\right)
$$


Apart from the definition of the innovation, modified as in (22), the update step is the same.

$$
z_{n}=Y_{n}-h\left(\hat{\chi}_{t_{n}}\right)
$$

The equations are the same than the ones in the cartesian case, see equations (14) to (19), except that step (17) is replaced by (22) and $\tilde{H}_{n}^{\text {cart }}$ is replaced by $\tilde{H}_{n}^{\text {pol }}$.

\section{Tracking application}

The aim is to apply the IEKF to target tracking. For the evolution model of the target, we use

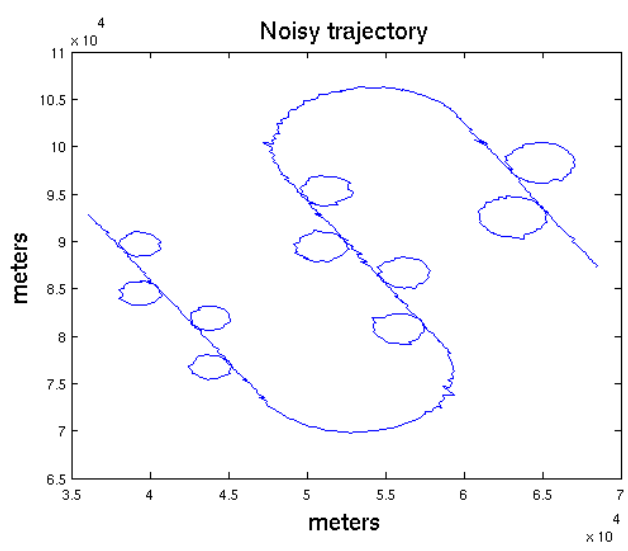

Figure 1: Trajectory followed by the target, with measurement noise

exactly the same model as presented above, expressed in intrinsic coordinates. We present the results of a numerical experiment on a synthetic trajectory inspired from a real fighter trajectory recorded by Thales. The measurements are in meters and give the cartesian coordinates, and the radar is centered at the origin. The measures were manually transformed in range and bearing coordinates with the function $h$. The measurement noise in range and bearing was also added by hand, as an additive noise.

The trajectory of the target is presented on Fig. 1. The amplitude of the measurement noise is visible on this figure. The results are presented for a measurement noise close to real measurement noises, and thus the measures are in polar coordinates. The angle $\theta$ is modulo $2 \pi$. On Fig. 2, the results are presented for an EKF with the same model as the one described for the IEKF, and on Fig. 3 the results are presented for the IEKF. The process noises are optimized for this particular trajectory by maximizing the measurement likelihood, as in [17]. Indeed, in the model, $\omega_{t}$ and $u_{t}$ are supposed to be constant, so we need to add some sufficiently high process noise to these variables to account for their variations. The RMSE for each parameter are presented on Tab. 1. We did three experiments: two for the IEKF, with hand-tuned process noises or optimized process noises, and one for the EKF with optimized process noises. The optimal noise tuning brings mostly better velocity and angle estimations, we could also optimize the tuning to favor some parameters, depending on the importance of each parameter. For the optimal tuning experiments, we see that the results for the IEKF are better than the results for the EKF, for all parameters. 

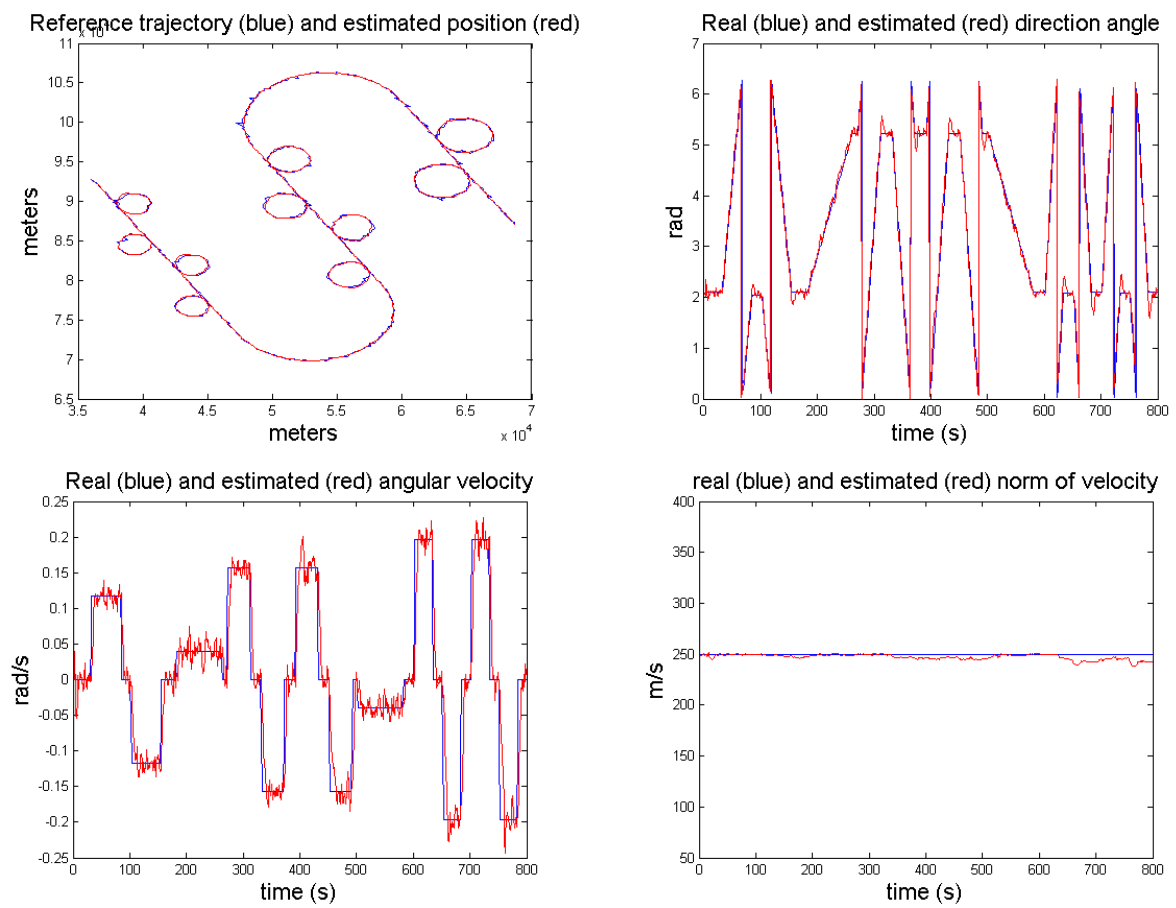

Figure 2: Trajectory (top left), $\theta$ (top right), $\omega$ (bottom left) and $u$ estimated for the EKF

\begin{tabular}{cccc}
\hline Parameter & Hand-tuned IEKF & Optimized IEKF noises & Optimized EKF noises \\
\hline$x^{(1)}$ & 36.6 & 34.2 & 36.2 \\
$x^{(1)}$ & 23.4 & 23.9 & 24.1 \\
$\theta(\mathrm{RMSE}$ for $1-\cos \theta)$ & 0.0097 & 0.0090 & 0.0099 \\
$\omega$ & 0.0221 & 0.0226 & 0.0226 \\
$u$ & 2.26 & 1.73 & 2.71 \\
\hline
\end{tabular}

Table 1: RMSE for each parameter on 100 Monte-Carlos and on the whole trajectory for a hand-tuned IEKF, an optimized IEKF and an optimized EKF

\section{Conclusion}

We have proposed an evolution model expressed in intrinsic coordinates, along with a filter that can cope with high nonlinearities in these particular evolution equations, this method is thus suitable for highly maneuvering targets. The filter is moreover compatible with realistic measurement noise. This study shows that the IEKF should be more stable than the EKF for other nonlinear systems that can be expressed in the same matricial framework, like targets with high lateral accelerations. The optimization of the noise tuning was done using only one trajectory. One should also compare results with one tuning for a bench of trajectories. To this aim, the tool MYRIAD, developed by Thales Research \& Technology has been developed, it is based on Choquet's integral [18]. This tool is designed to perform multi-criteria decision analysis to balance resources between algorithms, such as tracking algorithms and search patterns algorithms, as in [?]. Future work will also consist in developing further this method and in writing an 

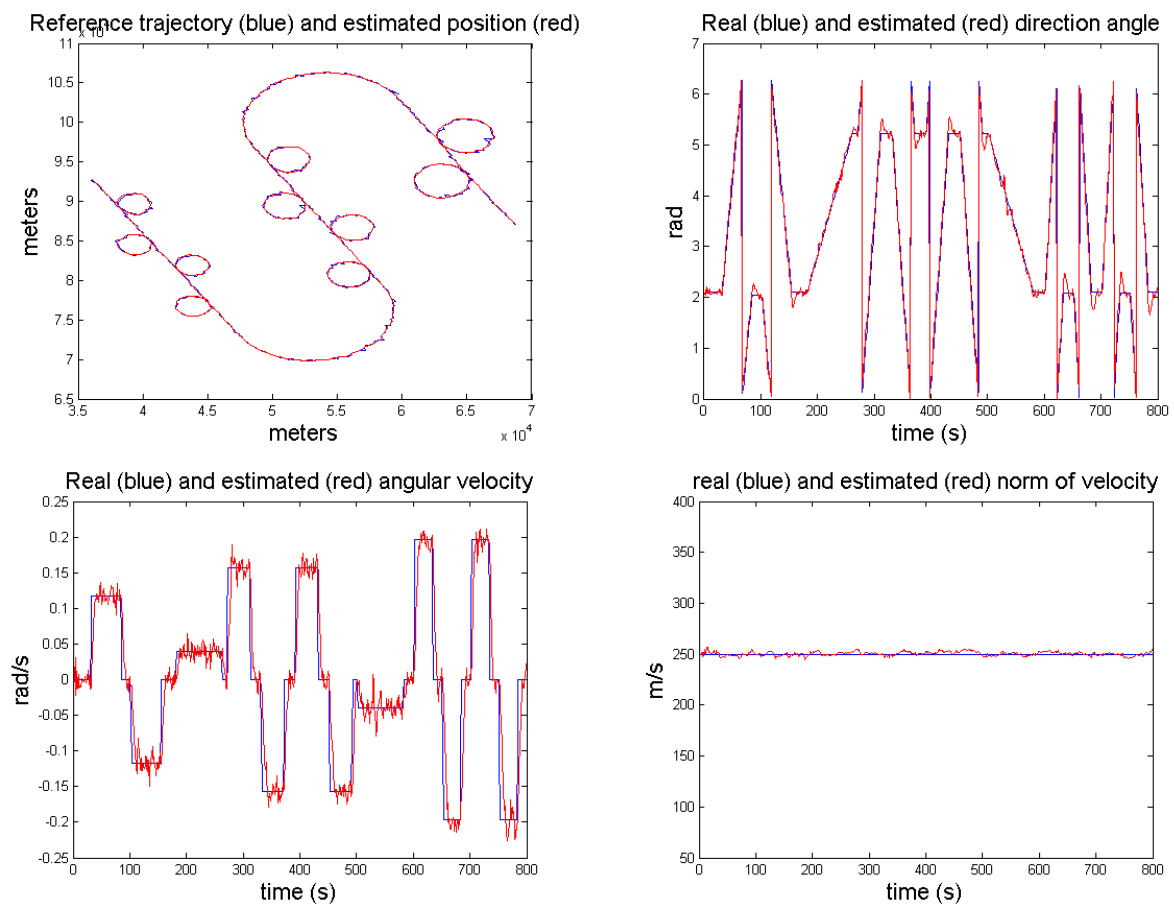

Figure 3: Trajectory (top left), $\theta$ (top right), $\omega$ (bottom left) and $u$ estimated for the optimized noises IEKF

Interacting Multiple Filter algorithm compatible with the Lie group structure used in the IEKF.

\section{Acknowledgement}

This work is partly supported by a DGA-MRIS scholarship.

\section{References}

[1] A. Barrau and S. Bonnabel, "Invariant particle filtering with application to localization," in Decision and Control (CDC), 2014 IEEE 53rd Annual Conference on. IEEE, 2014, pp. 5599-5605.

[2] V. Jeauneau, F. Barbaresco, and T. Guenais, "Radar tasks scheduling for a multifunction phased array radar with hard time constraint and priority," in International Radar Conference. IEEE, 2014.

[3] S. Godsill and J. Vermaak, "Variable rate particle filters for tracking applications," in IEEE/SP 13th Workshop on Statistical Signal Processing, 2005. IEEE, 2005, pp. 1280-1285.

[4] R. Frühwirth, "Application of kalman filtering to track and vertex fitting," Nuclear Instruments and Methods in Physics Research Section A, vol. 262, no. 2, pp. 444-450, 1987.

[5] S. J. Julier and J. K. Uhlmann, "Unscented filtering and nonlinear estimation," Proceedings of the IEEE, vol. 92, no. 3, pp. 401-422, 2004.

[6] F. Gustafsson, F. Gunnarsson, N. Bergman, U. Forssell, J. Jansson, R. Karlsson, and P.-J. Nordlund, "Particle filters for positioning, navigation, and tracking," IEEE Transactions on signal processing, vol. 50, no. 2, pp. 425-437, 2002. 
[7] Y. Bar-Shalom, K. Chang, and H. A. Blom, "Tracking a maneuvering target using input estimation versus the interacting multiple model algorithm," Aerospace and Electronic Systems, IEEE Transactions on, vol. 25, no. 2, pp. 296-300, 1989.

[8] F. Daum, "Nonlinear filters: beyond the kalman filter," IEEE Aerospace and Electronic Systems Magazine, vol. 20, no. 8, pp. 57-69, 2005.

[9] M. S. Grewal, Kalman filtering. Springer, 2011.

[10] L. Ljung, "Asymptotic behavior of the extended kalman filter as a parameter estimator for linear systems," IEEE Transactions on Automatic Control, vol. 24, no. 1, pp. 36-50, Feb 1979.

[11] M. Boutayeb, H. Rafaralahy, and M. Darouach, "Convergence analysis of the extended kalman filter used as an observer for nonlinear deterministic discrete-time systems," IEEE transactions on automatic control, vol. 42, no. 4, pp. 581-586, 1997.

[12] S. Bonnabel and J. J. Slotine, "A contraction theory-based analysis of the stability of the deterministic extended kalman filter," IEEE Transactions on Automatic Control, vol. 60, no. 2, pp. 565-569, Feb 2015.

[13] A. Barrau and S. Bonnabel, "The invariant extended kalman filter as a stable observer," IEEE Transactions on Automatic Control, 2017.

[14] _ - "Navigating with highly precise odometry and noisy GPS: a case study," in IFAC Proceedings Volumes. IEEE, 2016.

[15] R. E. Kalman and R. S. Bucy, "New results in linear filtering and prediction theory," Trans. ASME, Ser. D, J. Basic Eng, p. 109, 1961.

[16] R. M. Murray, Z. Li, and S. S. Sastry, A mathematical introduction to robotic manipulation. CRC press, 1994.

[17] P. Abbeel, A. Coates, M. Montemerlo, A. Y. Ng, and S. Thrun, "Discriminative training of kalman filters." in Robotics: Science and systems, vol. 2, 2005, p. 1.

[18] C. Labreuche, F. Barbaresco, D. N'Guyen, T. Guenais, and F. Gosselin, "Multi-criteria aggregation for adaptive multifunction radar resource management performances evaluation," submitted to 18th International Radar Symposium (IRS), 2017. 